\title{
ULTRA SHORT-TERM PREDICTION OF POLE COORDINATES VIA COMBINATION OF EMPIRICAL MODE DECOMPOSITION AND
}

\author{
NEURAL NETWORKS
}

\author{
Yu Lei ${ }^{123}$, Danning Zhao ${ }^{13}$, Hongbing Cai ${ }^{12}$ \\ ${ }^{1}$ National Time Service Center, Chinese Academy of Sciences, Xi'an 710600, China \\ ${ }^{2}$ Key Laboratory of Time and Frequency Primary Standards, Chinese Academy of Sciences, \\ Xi'an 710600, China \\ ${ }^{3}$ University of Chinese Academy of Sciences, Beijing 100049, China \\ E-mail: leiyu@ntsc.ac.cn
}

\begin{abstract}
It was shown in the previous study that the increase of pole coordinates prediction error for about 100 days in the future is mostly caused by irregular short period oscillations. In this paper, the ultra short-term prediction of pole coordinates is studied for 10 days in the future by means of combination of empirical mode decomposition (EMD) and neural networks (NN), denoted EMD-NN. In the algorithm, EMD is employed as a low pass filter for eliminating high frequency signals from observed pole coordinates data. Then the annual and Chandler wobbles are removed a priori from pole coordinates data with high frequency signals eliminated. Finally, the radial basis function (RBF) networks are used to model and predict the residuals. The prediction performance of the EMD-NN approach is compared with that of the $\mathrm{NN}$-only solution and the prediction methods and techniques involved in the Earth orientation parameters prediction comparison campaign (EOP PCC). The results show that the prediction accuracy of the EMD-NN algorithm is better than that of the NN-only solution and is also comparable with that of the other existing prediction method and techniques.
\end{abstract}

Keywords: pole coordinates, prediction, empirical mode decomposition (EMD), neural networks $(\mathrm{NN})$

\section{INTRODUCTION}

The Earth orientation parameters (EOP): universal time (UT1-UTC), $x_{p}, y_{p}$ pole coordinates and nutation-precession corrections $d \psi, d \varepsilon$, are demanded for various domains related to reference frames such as interplanetary spacecraft tracking and navigation, 
positional astronomy, geodesy and precise orbit determinations of artificial Earth satellites (Gambis and Luzum, 2011). Technically, they are the parameters which provide the transformation of the International Terrestrial Reference System (ITRS) to the International Celestial Reference System (ICRS) as a function of time. EOP derived from advanced space geodetic technologies, e.g., Global Navigation Satellite System (GNSS), Very Long Baseline Interferometry (VLBI), Satellite Laser Ranging (SLR), Doppler Orbitography and Radiopositioning Integrated by Satellite (DORIS), are available with a latency of several days due to the complexity of the data processing. Thus, regularly generated EOP predictions are published by several international and national services, e.g., the International Earth Rotation and Reference Systems Service (IERS), or the EOP Service of the Institute for Applied Astronomy (IAA) in Saint Petersburg, Russia (Malkin and Skurikhina, 1996).

EOP prediction is very complex to handle owing to the irregularities of EOP time series and hence it is theoretically much more rational to predict EOP by using neural networks (NN) since NN are very strong tools for forecasting stochastic and irregular signals (Zhang et al., 2012). It is proved that three-layer feed-forward NN can approximate any continuous function to any desired accuracy (Kurkova, 1992). NN require no a priori models in advance and thus it can avoid the model error and makes modeling of complicated time series quite feasible. NN have already been successfully applied to EOP prediction with accuracy equal to or even better than that of other prediction methods (Schuh et al., 2002; Kosek et al., 2005; Wang et al., 2008, 2014; Liao et al., 2012; Xu et al., 2012; Zhang et al., 2012; Lei et al., 2015). This paper focuses on predicting $x_{p}, y_{p}$ pole coordinates utilizing the $\mathrm{NN}$ technology.

It has been found that the increase of polar motion prediction error for about 100 days in the future is mostly caused by irregular short period oscillations, which is predominately excited by the equatorial components of atmospheric and oceanic angular momentum (AAM and OAM), and it is expected that those short period variations would not have as significant impacts on prediction error of pole coordinates (Kosek, 2011). The purpose of the present study is to supply accurate and rapid predictions of pole coordinates for 10 days in the future that are of significance for real-time and precision applications such as space navigation and precise orbit determinations of artificial Earth satellites. Since NN have high potential for prediction of quasi-periodic and irregular signals and empirical mode decomposition (EMD) is a forceful tool for separation of different frequency bands in time series, the integration of EMD into NN has been developed to enhance the prediction quality of polar motion. Here, EMD is employed as a data pre-processing tool to remove high frequency signals from the observed time series of pole coordinates mainly because of its merit of adaptive decomposition. After removal of high frequency signals, the pre-processed data are forecasted by means of radial basis function (RBF) neural networks.

This paper is divided into four sections. Following the introduction, Section 2 reviews EMD and RBF network algorithms and describes the methodology for predicting pole coordinates based on the combination of EMD and RBF networks. The results predicted by the proposed hybrid method are analyzed and compared with those obtained by other prediction approaches in Section 3, followed by summary and conclusions in Section 4. 


\section{METHODOLOGY}

\subsection{EMPIRICAL MODE DECOMPOSITION (EMD)}

EMD can be an important tool for analyzing non-linear and non-stationary time series. It has some advantages over wavelet decomposition (Huang et al., 1998, 2003). Firstly, EMD is easy to understand and use. Secondly, EMD dose not need to use a mother function unlike wavelet decomposition. Most important of all, EMD is a fully data-driven tool for reducing any given data into a collection of intrinsic mode functions (IMF). In other words, EMD can adaptively decompose a signal into several independent fluctuation components according to local characteristics of a signal.

The basic principle of EMD is to decompose raw time series into a finite and small number of oscillatory functions, namely IMF. These IMF have to satisfy the following two prerequisites (Huang et al., 1998, 2003).

(1) In whole data set, the total number of local maxima and minima and the number of zero crossings must either be equal or differ at most by one, and

(2) the mean value of the envelope defined by local maxima and minima must be zero at all points.

The essence of EMD is a sifting procedure which extracts IMF from an original signal. The EMD algorithm can be described as follows (Huang et al., 1998, 2003).

Step 1: Identify all local maxima and minima in raw time series $s(t)(t=1,2, \cdots, l)$.

Step 2: Connect all the local maxima by a cubic spline line as the upper envelope $e_{\text {up }}(t)$. Repeat the procedure for the local minima to produce the lower envelope $e_{\mathrm{low}}(t)$.

Step 3: Compute the mean value of the point-by-point envelope from the upper and lower envelope and then derive the mean envelope $m(t)$ by the formula

$$
m(t)=\left[e_{\text {up }}(t)+e_{\text {low }}(t)\right] / 2,
$$

Step 4: Calculate the difference between the mean envelope $m(t)$ and original time series $s(t)(t=1,2, \cdots, l)$, i.e.,

$$
h(t)=s(t)-m(t),
$$

Step 5: Check whether the proto-intrinsic mode function $h(t)$ conforms to the property of IMF. If so it is regarded as the $i$ th IMF and then the residuals $r(t)=s(t)-h(t)$ take the place of the original time series $s(t)(t=1,2, \cdots, l)$. If not, $s(t)$ is replaced by $h(t)$. 
Step 6: Steps 1-5 are repeated until the size of the standard deviation calculated from the two consecutive sifting results is smaller than the pre-determined threshold.

By using the above-described sifting process, a collection of IMF can be obtained from high frequency to low frequency and accordingly the original time series $s(t)(t=1,2, \cdots, l)$ can be decomposed into a set of IMF and one residual as

$$
s(t)=\sum_{i=1}^{n} c_{i}(t)+r_{n}(t)
$$

where $n$ is the number of IMF, $r_{n}(t)$ is the residual trend and $c_{i}(t)(i=1,2, \cdots, n)$ represents IMF that are nearly orthogonal to each other and periodic. Each IMF is independent and specific for expressing local characteristics of an original signal.

\subsection{RADIAL BASIS FUNCTION (RBF) NEURAL NETWORKS}

RBF neural networks typically have three layers: an input layer, a hidden layer with a non-linear RBF activation function and a linear output layer (Park and Sandberg, 1991). The input can be modeled as a vector of real numbers $x \in \mathrm{R}^{u}$. The network output is then a scalar function of the input vector, $\varphi: \mathrm{R}^{u} \rightarrow \mathrm{R}^{v}$, and is given by

$$
\varphi(\boldsymbol{x})=\sum_{i=1}^{m} w_{i} \phi\left(\left\|\boldsymbol{x}-\boldsymbol{c}_{i}\right\|\right)
$$

where $m$ is the number of neurons in the hidden layer, $w_{i}$ is the weight of $i$ th neuron in the linear output neuron, $c_{i}$ is the center vector for $i$ th neuron in the hidden layer, and $\|g\|$ and $\phi(g)$ represent the Euclidean distance and the RBF, respectively. Functions that rely only on the distance from a center vector are radially symmetric about that vector, hence the name $\mathrm{RBF}$. In the basis form all inputs are connected to each hidden neuron. The RBF is commonly taken to be Gaussian:

$$
\phi\left(\left\|\boldsymbol{x}-\boldsymbol{c}_{i}\right\|\right)=\exp \left(-\frac{\left\|\boldsymbol{x}-\boldsymbol{c}_{i}\right\|^{2}}{2 \sigma_{i}^{2}}\right),
$$

where $\sigma_{i}$ is the width of the $i$ th RBF. The parameters $w_{i}, \boldsymbol{c}_{i}$ and $\sigma_{i}$ can be determined in a manner that optimizes the fit between the network output and the target output.

RBF networks are typically trained by a two-step algorithm. In the first step, the center vector $c_{i}$ is chosen. This step can be performed in several ways. For instance, $c_{i}$ can be randomly sampled from some set of samples or it can be determined using k-means clustering. 
The second step fits a model with coefficients $w_{i}$ and $\sigma_{i}$ with respect to some objective functions such as the least squares (LS) function. In this paper, the orthogonal least square (OLS) method is employed for selecting the center vector $c_{i}$ and determining the parameters $w_{i}$ and $\sigma_{i}$ (Chen et al., 1991). In the OLS method the Gram-Schmidt algorithm and adaptive gradient descent procedure are used for selection of the center vector $c_{i}$ and adaption of the parameters $w_{i}$ and $\sigma_{i}$, respectively. The parameters $w_{i}$ and $\sigma_{i}$ can be obtained by minimizing the following objective function

$$
\min J=\sum_{i=1}^{v}\left|y_{\mathrm{n} i}-y_{\mathrm{di}}\right|^{2}
$$

where $y_{\mathrm{n} i}$ and $y_{\mathrm{d} i}$ are the network output and the desired target output for the $i$ th neuron in output layer, respectively.

\subsection{EMD-NN PREDICTION METHOD}

For the prediction of pole coordinates, a hybrid scheme is developed as depicted in the flowchart of Figure 1, represented as the EMD-NN. It comprises three main stages. In the first stage, observed data of pole coordinates is pre-processed where EMD is employed to decompose raw time series into a small number of IMF and then those IMF corresponding to the high frequency components of pole coordinates are removed from original data. The second stage involves generation of the a priori model to extract the seasonal fluctuations including the annual and Chandler wobbles and derive the residuals of pole coordinates. In the third stage, a NN model is composed based on the derived residuals. The subsequently predicted residuals are then added to the a priori model to obtain the forecasted values of pole coordinates.

The following three sections briefly explain the methodology for predicting pole coordinates, including implementation of EMD filtering procedure, generation of the a priori model and composition of the training patterns. 


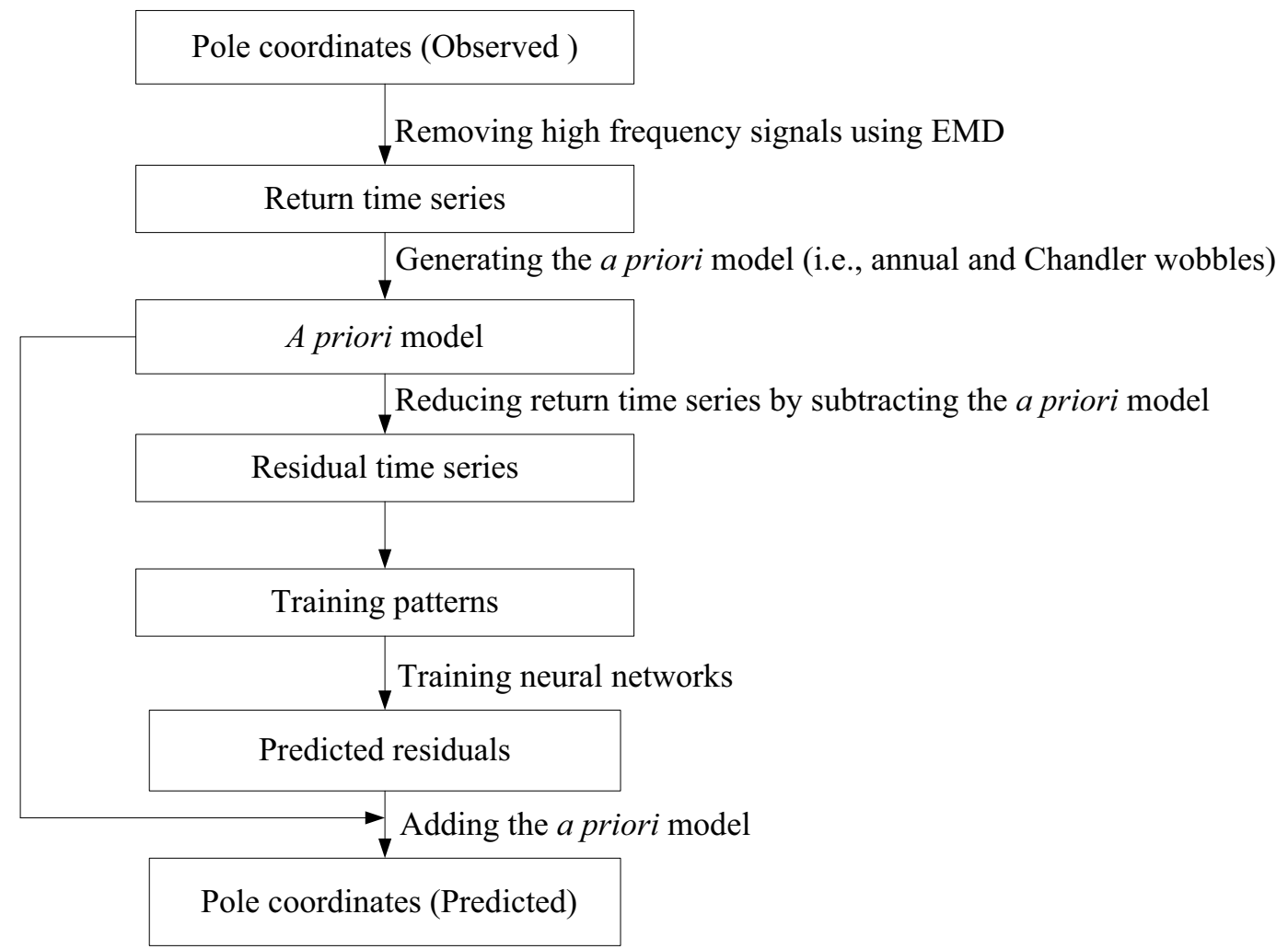

Fig. 1. The EMD-NN method for polar motion prediction.

\subsubsection{DATA PRE-PROCESSING USING EMD}

It have been proved that EMD can act as a filter bank of low pass, high pass or band pass (Flandrin et al., 2004). In this work, EMD is essentially used as a filter bank of high pass to remove high frequency signals from observed pole coordinates resembling wavelet decomposition. Observed pole coordinates data is first decomposed into a finite number of IMF by the EMD algorithm. Next those first generated IMF corresponding to very high frequency components highly correlated with AAM and OAM are removed from the raw time series, and then the return time series $s^{\prime}(t)(t=1,2, \cdots, l)$ are derived from

$$
s(t)=s^{\prime}(t)-\sum_{i=1}^{h} c_{i}(t)
$$

where $c_{i}(t)(i=1,2, \cdots, u)$ are those IMF representing high frequency components in original time series, and $U$ has to be selected according to a matter of experience.

\subsubsection{GENERATION OF THE $A$ PRIORI MODEL}

Observed time series of pole coordinates consist of a linear trend and some seasonal fluctuations such as the annual and Chandler wobbles. In order to avoid the error coming from the extrapolation problem, a linear part plus seasonal oscillations are reduced from filtered data of polar motion. To this end the parameters of a linear trend and seasonal variations are estimated by the LS approach from filtered pole coordinates data: bias $\left(a_{0}\right)$ and $\operatorname{drift}\left(a_{1}\right)$ of 
the linear part, amplitudes $\left(A_{\mathrm{aw}}, A_{\mathrm{cw}}\right)$ and phases $\left(\Phi_{\mathrm{aw}}, \Phi_{\mathrm{cw}}\right)$ of the annual and Chandler wobbles. For completeness, the a priori model can therefore written as

$$
f_{\mathrm{PM}}(t)=a_{0}+a_{1} t+A_{\mathrm{aw}}\left(\omega_{\mathrm{aw}} t+\Phi_{\mathrm{aw}}\right)+A_{\mathrm{cw}}\left(\omega_{\mathrm{cw}} t+\Phi_{\mathrm{cw}}\right)
$$

where $\omega_{\mathrm{aw}}=2 \pi / 365.24$ and $\omega_{\mathrm{cw}}=2 \pi / 432.08$. This model is used to derive the polar motion residuals using the equation $\xi(t)=s^{\prime}(t)-f_{\mathrm{PM}}(t)$. In the process of predictions, extrapolation of the a priori model is utilized to calculate the deterministic predictions of pole coordinates.

\subsubsection{COMPOSITION OF THE TRAINING PATTERNS}

After time series of pole coordinates have been reduced, the training patterns are composed for prediction days in the future based on the residuals $\xi(t)(t=1,2, \cdots, l)$. Considering the fact that the closer the observational data is to the day to be forecasted, the greater influence on the prediction is, we form the training patterns as bellows where the residuals of the last 1 , 2, 3, 4 and 5 days are used to extrapolate the residual of the next day

$$
\begin{array}{cc}
\{\xi(t-5), \xi(t-4), \xi(t-3), \xi(t-2), \xi(t-1)\} & \xi(t) \\
\downarrow & \downarrow \\
\text { input vector } & \text { output }
\end{array} .
$$

Unlike conventional training patterns, the patterns utilize predicted values as inputs in already existing models to compute the corresponding prediction values for the future days. The pattern matrices are then shifted along whole residual time series to construct a multitude of pattern pairs.

\section{PREDICTION RESULTS AND COMPARISON WITH OTHER METHODS}

\subsection{DATA DESCRIPTION}

The IERS publishes daily values of EOP series (EOP C04) with a latency of several days after the processing of observations of all advanced space geodetic techniques (GNSS, VLBI, SLR and DORIS) gathered at the receivers of permanent ground stations distributed around the world. The EOP series are estimated together with the station coordinates and velocities from each technique. The subsequently estimated EOP from all the above-mentioned techniques are then combined to derive the final EOP solutions. These finally combined EOP solutions are regularly published at the IERS website (ftp://hpiers.obspm.fr/iers/eop/eopc04/eopc04_IAU2000.62-now). In this contribution, the IERS EOP C04 series are used as data base. 


\subsection{NUMERICAL EXAMPLES AND RESULTS}

The predictions based on the EMD-NN method and the NN-only method have been calculated. The NN-only scheme employs raw time series of pole coordinates without the EMD filtering process and uses same training patterns as the EMD-NN method.

The EOP 08C04 series with daily values from the IERS between January 1, 2004 and July 15, 2015 are used for the modeling and evaluation of the EMD-NN and NN-only models. The data of a length of 10a between January 1, 2004 and December 31, 2013 were used to build the prediction models while the remaining data from January 1, 2014 to July 15, 2015 to validate the composed models. The observed time series of pole coordinates are first decomposed and the three stochastic and irregular high frequency components $\left(c_{1}, c_{2}\right.$ and $\left.c_{3}\right)$ whose periods are shorter than one day are then removed (i.e., $u=3$ in Equation 7). As an instance, the decomposition of the time series of the $y_{p}$ polar motion component spanning from January 1, 2004 to December 31, 2013 is shown in Figure 2. Next the training patterns are composed and then the EMD-NN and NN-only models are built based on the return signal $s^{\prime}(t)$ and the raw signal $s(t)$, respectively. After the models have been set up, the composed models are used to produce a predicted set of pole coordinates for the future 1-360 days. Figure 3 and Table 1 illustrate the results comparing the accuracy of the predictions by the EMD-NN and NN-only methods, where the root mean squares error (RMSE) is computed for the $i$ th day in the future as follows.

$$
\operatorname{RMSE}_{p}=\sqrt{\frac{1}{k} \sum_{i=1}^{k}\left(O_{p}^{k}-F_{p}^{k}\right)^{2}},
$$

where $F_{p}$ represents the forecasted value of the composed models for the prediction day $p$, $O_{p}$ denotes the corresponding observed value of the IERS 08C04 series and $k$ is the number of predictions made for the particular prediction day. 200 predictions starting at different days have been made for each prediction day to calculate the RMSE i.e. $k=200$.

From Figure 3 and Table 1 it can be seen that the results of predictions for 10 days in the future obtained by the developed EMD-NN algorithm are more accurate than those by the $\mathrm{NN}$-only strategy. For the predictions of the $x_{p}$ component of pole coordinates, the accuracy of predictions by the EMD-NN approach improves by about $22.09 \%$ at most and about $10.69 \%$ on average in contrast to that by the NN-only method in terms of RMSE. For the $y_{p}$ component of polar motion, the prediction accuracy based on the EMD-NN scheme enhances by about $18.22 \%$ at most and about $7.79 \%$ on average compared with the accuracy of the direct NN predictions. What can be concluded with the information available from the numerical results is that the prediction error of pole coordinates obtained after removing three frequency components with the highest frequencies are noticeably smaller than the prediction 
error of raw pole coordinates data at least for a few days in the future. In other words, if pole coordinates data are composed of the annual, Chandler and longer periodic variations then the prediction error becomes very small. It means that the short periodic variations have significant influences on the prediction error of polar motion.
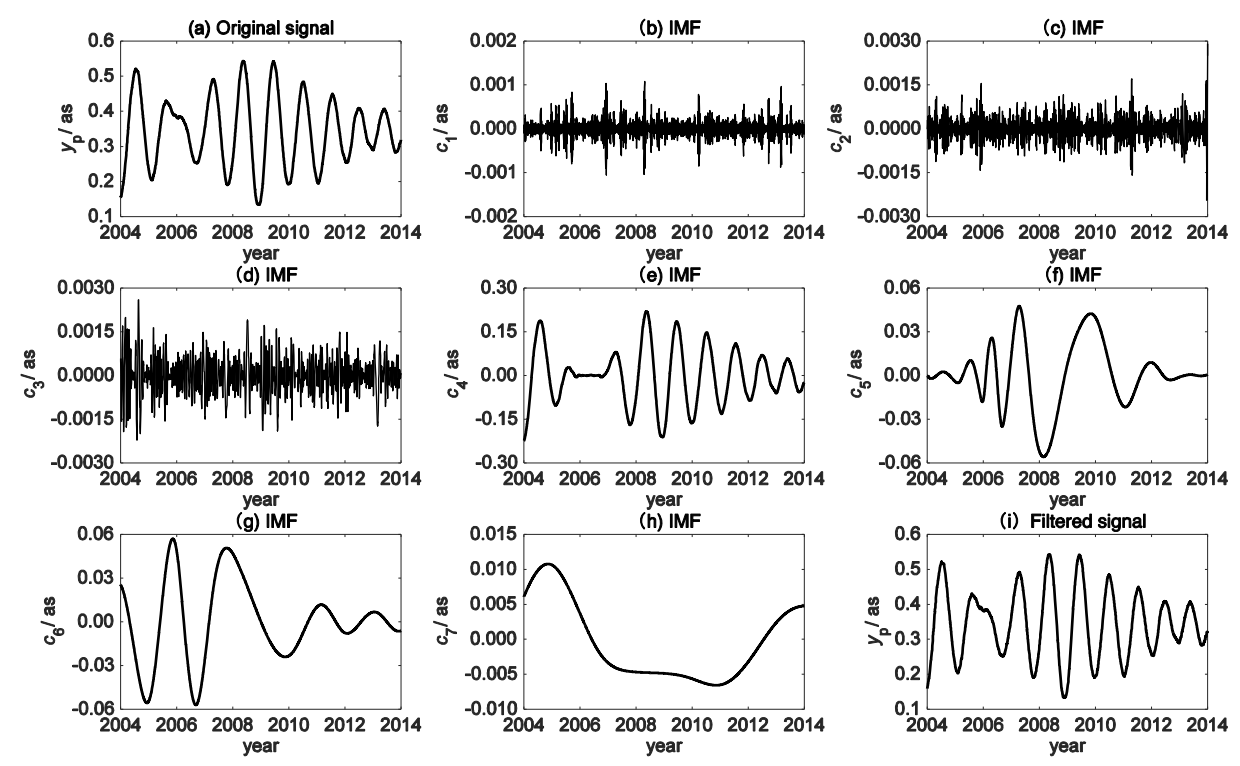

Fig. 2. Original, decomposed and filtered time series of the $y_{p}$ polar motion component.
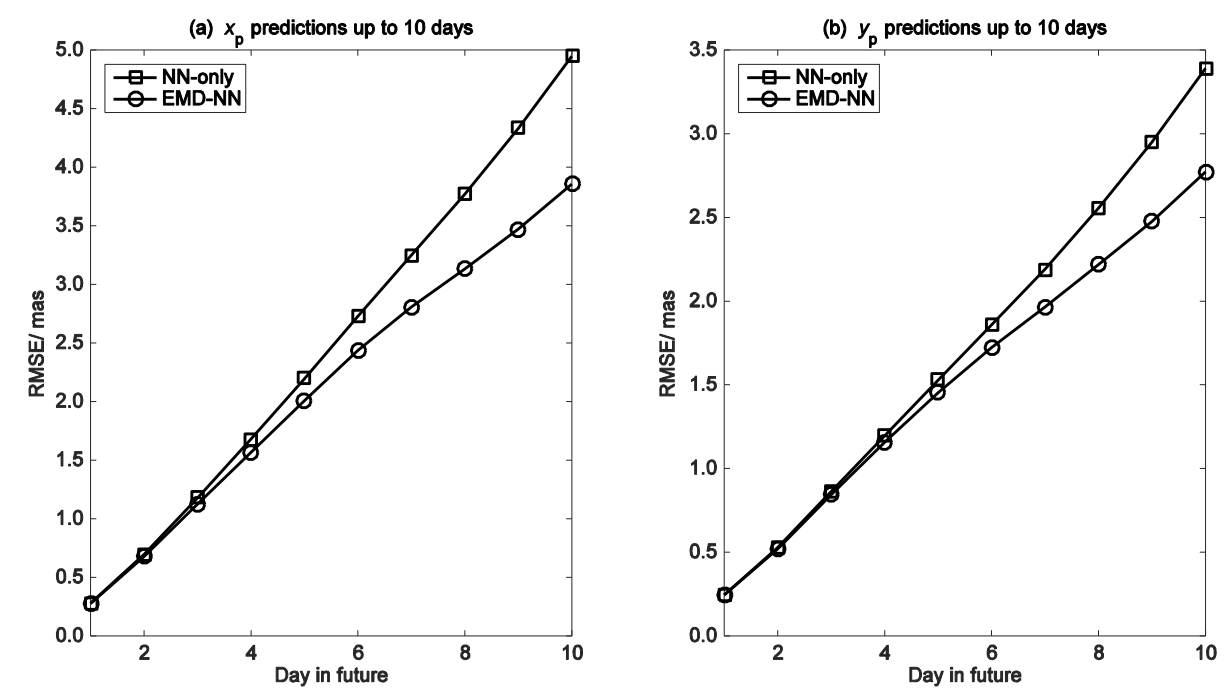

Fig. 3. RMSE of ultra short-term (1 10 days) predictions of pole coordinates by the EMD-NN and NN-only methods. 
Table 1. RMSE for different prediction lengths of pole coordinates by the EMD-NN and NN-only methods

\begin{tabular}{ccccccc}
\hline Prediction day & \multicolumn{2}{c}{ NN-only } & \multicolumn{3}{c}{ EMD-NN } & \multicolumn{2}{c}{ Improvement } \\
& $x_{p}[\mathrm{mas}]$ & $y_{p}[\mathrm{mas}]$ & $x_{p}$ [mas] & $y_{p}$ [mas] & \multicolumn{1}{c}{$x_{p}$} & $y_{p}$ \\
\hline 1 & 0.279 & 0.246 & 0.275 & 0.244 & $1.43 \%$ & $0.81 \%$ \\
2 & 0.696 & 0.528 & 0.677 & 0.519 & $2.73 \%$ & $1.70 \%$ \\
3 & 1.179 & 0.863 & 1.126 & 0.844 & $4.50 \%$ & $2.20 \%$ \\
4 & 1.680 & 1.196 & 1.569 & 1.157 & $6.61 \%$ & $3.26 \%$ \\
5 & 2.197 & 1.529 & 2.010 & 1.454 & $8.51 \%$ & $4.91 \%$ \\
6 & 2.726 & 1.858 & 2.435 & 1.723 & $10.68 \%$ & $7.27 \%$ \\
7 & 3.248 & 2.190 & 2.807 & 1.965 & $13.58 \%$ & $10.27 \%$ \\
8 & 3.771 & 2.556 & 3.133 & 2.219 & $16.92 \%$ & $13.18 \%$ \\
9 & 4.332 & 2.950 & 3.471 & 2.475 & $19.88 \%$ & $16.10 \%$ \\
10 & 4.948 & 3.391 & 3.855 & 2.773 & $22.09 \%$ & $18.22 \%$ \\
\hline
\end{tabular}

In order to furthermore assess the performance of the presented EMD-NN prediction model, the results of this model are also compared with those of the Earth orientation parameters prediction comparison campaign (EOP PCC) lasting from October 1, 2005 to February 28, 2008 , in which the prediction period and validation scheme had been clearly specified in advance (Kalarus et al., 2008, 2010). Using the well-defined rules a comparison with other prediction methods and techniques participating in the EOP PCC is shown in Figure 4, where the prediction period is the same, the statistics are referred to the IERS EOP 05 C04 series and the mean absolute error (MAE) is used as the statistical measure computed by the following

$$
\operatorname{MAE}_{p}=\frac{1}{k} \sum_{i=1}^{k}\left|O_{p}^{k}-F_{p}^{k}\right|
$$

From the comparison it can be said that the accuracy of the predictions for $1 \sim 5$ days in the future by the EMD-NN model is equal to or even better than the prediction accuracy of the most accurate prediction technique for polar motion forecast, namely the LS extrapolation of the harmonic model and autoregressive prediction. Since the $6^{\text {th }}$ day, the prediction error of the EMD-NN model gradually increases and is much bigger than that of the most accurate prediction technique, but is comparable with that of the other existing prediction approaches yet. It should be noted that in the real-time day-to-day competition real-time EOP C04 estimates for the last days are used, while in our prediction the more precise re-estimated values are utilized. Thus in spite of using the same equation for the computing MAE and same EOP reference series (EOP 05 C04 of IERS), this might have influenced the results of the other participants. 

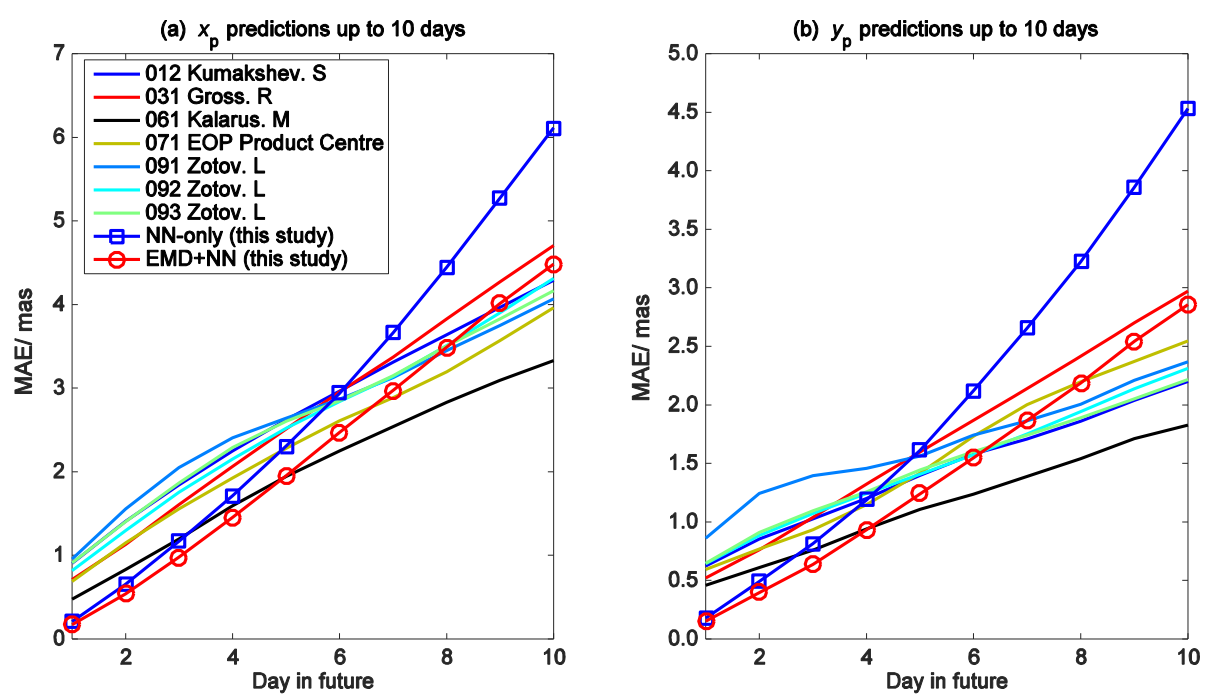

Fig. 4. Comparison of MAE prediction errors of different prediction methods and techniques.

\section{SUMMARY AND CONCLUSIONS}

It has been revealed that prediction error of pole coordinates for about 100 days in the future is mostly caused by the wideband short period fluctuations with periods less than half a year in the previous research. These high frequency variations in pole coordinates data are predominately excited by the equatorial components of AAM and OAM. In order to reduce the contribution of the irregular short period oscillations to prediction error of pole coordinates, these wideband signals with short periods have to be removed from observed time series of pole coordinates. In the present study, a hybrid model called the EMD-NN method is recommended for prediction of pole coordinates data, in which EMD is used for subtracting the components with highest frequencies from the original time series. It has been shown that EMD can be employed to filter those high frequency stochastic signals from pole coordinates data. Additionally, direct $\mathrm{NN}$ predictions are made with the same data set to perform an objective comparison. The architecture of the NN model has been kept the same for both prediction methods. Individual prediction approaches were realized for the prediction of specific days in the future.

The results show that the ultra short-term prediction error for 10 days in the future is much smaller when pole coordinates data are smoothed by removing the components with short periods using the multi-scaling nature of EMD, demonstrating the contribution of high frequency signals to prediction errors of polar motion. The combined EMD-NN prediction scheme performs better than the NN-only method using raw polar motion data at least for ultra short-term prediction. In addition, the comparison with the EOP PCC results illustrates that the prediction accuracy is comparable with the existing prediction methods and techniques. So the presented EMD-NN prediction method is an appropriate tool for predicting pole coordinates data. The last but not least conclusion is that the short period fluctuations in pole coordinates data should not been involved in prediction algorithms and should be removed from original data before modeling since the prediction error increase is indeed caused by those high frequency oscillations. 
ACKNOWLEDGEMENTS. The authors are grateful to the IERS for providing pole coordinates data. The authors also thank Kalarus M. for providing the EOP PCC data. This work is supported by the West Light Foundation of Chinese Academy of Sciences.

\section{REFERENCES}

Chen, S., Cowan, C.F.N. and Grant, P.M. (1991). Orthogonal Least Squares Learning Algorithm for Radial Basis Function Networks. IEEE Transactions on Neural Networks, Vol. 2, No. 2, 302-309.

Flandrin, P., Rilling, G. and Goncalvés, P. (2004). Empirical Mode Decomposition as a Filter Bank, IEEE SIGNAL PROCESSING LETTERS, Vol. 11, No. 2, 112-114.

Gambis, D. and Luzum, B. (2011). Earth Rotation Monitoring, UT1 Determination and Prediction. Metrologia, Vol. 48, No. 4, 165-170.

Huang, N.E., Shen, Z., Long, S.R., Wu, M.C., Shih, H.H., Zheng, Q., Yen, N.C., Tung, C.C. and Liu, H.H. (1998). The Empirical Mode Decomposition and the Hilbert Spectrum for Nonlinear and Non-Stationary Time Series Analysis. Proceedings of the Royal Society London: A, Vol. 454, 903-995.

Huang, N.E., Wu, M.L., Qu, W., Long, S.R. and Shen, S.S.P.H. (2003). Application of Hilbert-huang Transform to Non-Stationary Financial Time Series Analysis. Applied Stochastic Models in Business and Industry, Vol. 19, No. 3, 245-268.

Kalarus, M., Kosek, W., Schuh, H. (2008). Summary of the Earth Orientation Parameters Prediction Comparison Campaign. EGU General Assembly 2008, EGU abstract: EGU2008-A-00595.

Kalarus, M., Schuh, H., Kosek, W., Akyilmaz, O. and Bizouard, Ch. (2010). Achievements of the Earth Orientation Parameters Prediction Comparison Campaign. Journal of Geodesy, Vol. 84, No. 10, 587-596.

Kosek, W. (2011). Future Improvements in EOP Prediction. Geodesy for Plant Earth, International Association of Geodesy Symposia, Vol. 136, 513-520.

Kosek, W., Kalarus, M., Johnson, T.J., Wooden, W.H., McCarthy, D.D. and Popiński, W. (2005). A Comparison of LOD and UT1-UTC Forecasts by Different Combined Prediction Techniques. Artificial Satellites, Vol. 40, No. 2, 119-125.

Kurkova, V. (1992). Kolmogorov's Theorem and Multilayer Neural Networks. Neural Networks, Vol. 5, No. 3, 501-506.

Lei, Y., Zhao, D.N. and Cai, H.B. (2015). Extreme Learning Machines for the Predictions of Length of Day. Artificial Satellites, Vol. 50, No. 1, 19-33.

Liao, D.C., Wang, Q.J., Zhou, Y.H., Liao, X.H. and Huang, C. L. (2012). Long-term Prediction of the Earth Orientation Parameters by the Artificial Neural Network Technique. Journal of Geodynamics, Vol. 62, No. 8, 87-92.

Park, J. and Sandberg, I.W. (1991). Universal Approximation Using Radial-Basis-Function Networks. Neural Computing, Vol. 3, No. 2, 246-257. 
Schuh, H., Ulrich, M., Egger, D., Müller, J. and Schwegmann, W. (2002). Prediction of Earth Orientation Parameters by Artificial Neural Networks. Journal of Geodesy, Vol. 76, No. 5, 247-258.

Wang, Q.J., Du, Y.N. and Liu, J. (2014). Introducing Atmospheric Angular Momentum into Prediction of Length of Day Change by Generalized Regression Neural Network Model. Journal of Central South University, Vol. 21, No. 4, 1396-1401.

Wang, Q.J., Liao, D.C. and Zhou, Y.H. (2008). Real-Time Rapid Prediction of Variations of Earth's Rotational Rate. Chinese Science Bulletin, Vol. 53, No. 7, 969-973.

$\mathrm{Xu}$, X.Q., Zotov, L. and Zhou, Y.H. (2012). Combined Prediction of Earth Orientation Parameters. China Satellite Navigation Conference (CSNC) 2012 Proceedings Lecture Notes in Electrical Engineering, Vol. 160, No. 2, 361-369.

Zhang, X.H., Wang, Q.J., Zhu, J.J. and Zhang, H. (2012). Application of General Regression Neural Network to the Prediction of LOD Change. Chinese Astronomy and Astrophysics, Vol. 36, No. 1, 86-96.

Received: 2016-06-22,

Reviewed: 2016-10-18, by L. Zotov, and 2016-10-21,

Accepted: 2016-11-07. 